\title{
Archéopages
}

Archéopages Archéologie et société

34 | 07/2012

Campagnes

\section{Industries orléanaises}

\section{Gregory Vacassy}

\section{OpenEdition}

Journals

Édition électronique

URL : https://journals.openedition.org/archeopages/431

DOI : $10.4000 /$ archeopages.431

ISSN : 2269-9872

\section{Éditeur}

INRAP - Institut national de recherches archéologiques préventives

\section{Édition imprimée}

Date de publication : 1 février 2012

Pagination : 106-107

ISSN : 1622-8545

\section{Référence électronique}

Gregory Vacassy, «Industries orléanaises », Archéopages [En ligne], 34 | 07/2012, mis en ligne le 01 juillet 2012, consulté le 22 janvier 2022. URL : http://journals.openedition.org/archeopages/431 ; DOI : https://doi.org/10.4000/archeopages.431 
Période

Époque moderne

\section{Industries orléanaises}

Grégory Vacassy

Inrap, responsable d'opération

Site

La Motte Sanguin

Orléans

Loiret

Date

16 mai-o8 juillet 2011

Surface fouillée

Environ $800 \mathrm{~m}$

Équipe

Étude documentaire

Pascal Poulle, Inrap
L'emprise de l'étude se concentre sur une terrasse d'artillerie construite dans la première moitié du Xvi ${ }^{\mathrm{e}}$ siècle au pied de la courtine, bâtie quant à elle dans la seconde moitié du siècle précédent, et sur le fossé creusé en avant. Si l'histoire militaire de la ville est par la suite marquée par deux sièges lors des guerres de religion en 1562 et 1567 , les fortifications perdent ensuite rapidement leur utilité et sont déjà en partie détruites à la fin du XVIII ${ }^{\mathrm{e}} \mathrm{s}$. (Jesset, 2005).

C'est donc sur un vaste terrain démilitarisé qu'est transférée, dans les années 1790-1793, une manufacture de coton située jusque-là en ville (Robert, 2008) [ill. 1] Les bureaux sont installés dans l'ancienne tour d'angle de la Brebis et la caponnière attenante est surélevée pour l'occasion. La filature, construite sur sept étages, est orientée nord-sud et s'étend sur toute la largeur de la terrasse d'artillerie. Ainsi, elle prend appui, à l'est, sur l'imposant mur de soutènement de cette terrasse (conservé sur cinq mètres d'épaisseur et autant de profondeur), et, à l'ouest, sur la courtine. Une série de piliers maçonnés, dont deux ont été dégagés lors de la fouille, sert de point d'appui intermédiaire entre les deux murs gouttereaux. Afin d'actionner l'ensemble des outils de production, une «pompe a feu » puise l'eau dans la Loire qui baigne alors le sud du site, et la transforme en vapeur à l'aide d'une imposante chaudière [ill. 2].

Après bien des vicissitudes l'entreprise fait faillite en 1808 . En 1823 c'est une minoterie qui s'installe dans les locaux de l'ancienne usine avec douze moulins dit «américains » qui sont actionnés par une nouvelle pompe à feu. La méthode américaine consiste à supprimer toute opération manuelle, depuis la livraison du grain jusqu'à l'expédition des farines (Daumas, 1980, p. 220). Mais la production ne dure que peu de temps et semble même stoppée dès l'année suivante.

Cette activité de meunerie reprend à la fin des années 1850 sous la houlette de plusieurs associés. Le projet comprend en outre un atelier de panification. Dans la nuit du 19 juillet 1858 , un violent incendie
$\stackrel{N}{\Lambda}$

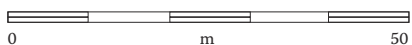

Cadastre

Bâtiments actuels

$-\neg$ Emprise de la fouille

Fortifications $\mathrm{Xv}^{\mathrm{e}}-\mathrm{XVI}^{\mathrm{e}}$ siècle

Fossé de ville

Emprise de la manufacture de coton

Vestiges de la manufacture de coton

Bâtiment appartenant à la minoterie, maçonnerie conservée

Bâtiment appartenant à la minoterie, maçonnerie récupérée

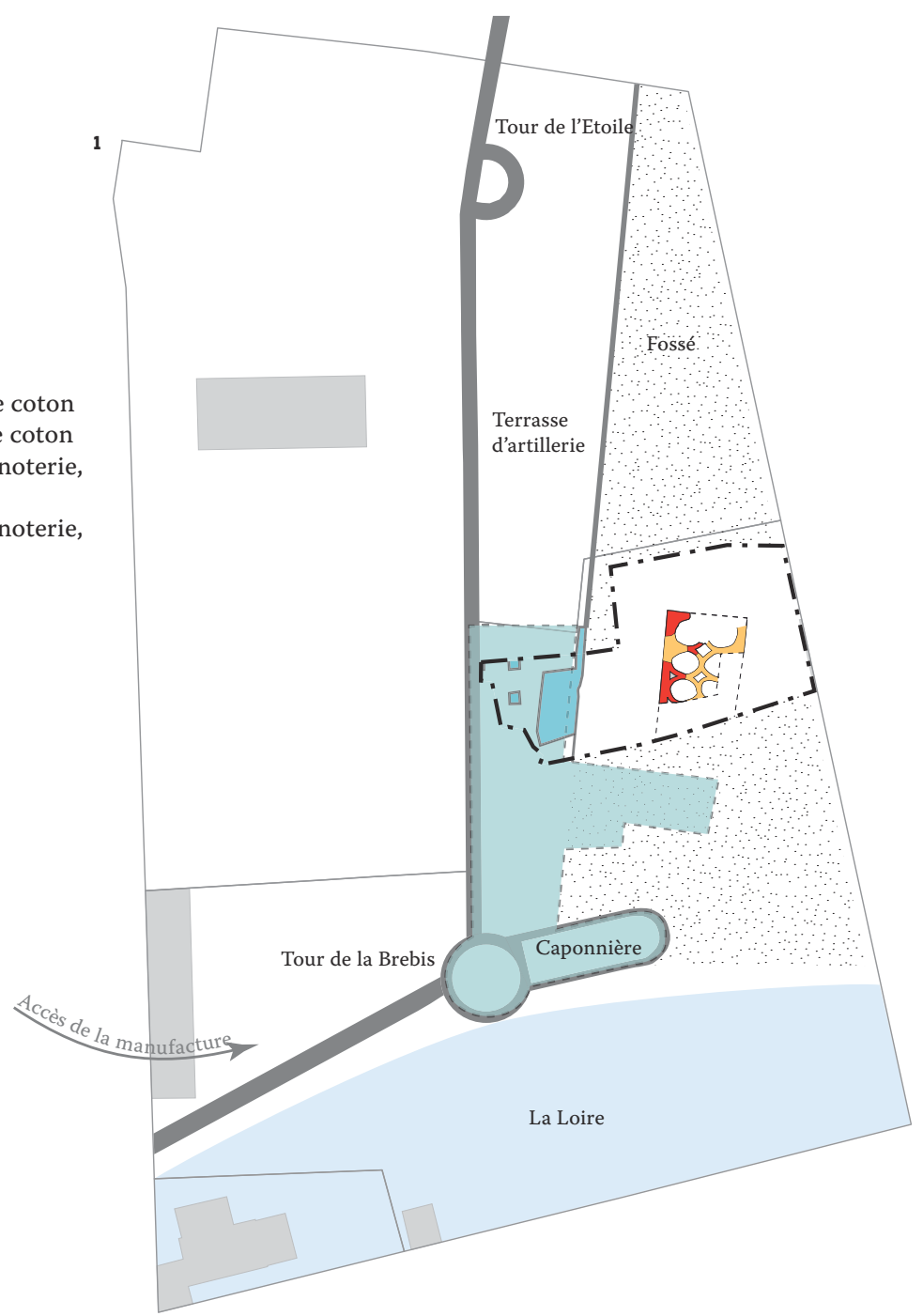


endommage très sérieusement les locaux, au moment où le projet est sur le point d'aboutir. Les documents concernant les travaux de remise en état indiquent que «neufs paires de meules » sont situées au rez-dechaussée du bâtiment de la manufacture, mais ils ne précisent pas où sont situés les fours destinés à cuire la production. Ceux-ci, à air chaud et sole tournante, présentent des améliorations techniques dévoilées lors de l'exposition universelle de 1855 . Un second incendie, en 1860, provoque l'arrêt définitif de l'activité.

C'est à cette deuxième minoterie que doit être rattachée une construction dégagée sur une longueur de 12,50 m et à là largeur de 10,70 m. Orientée nord-sud et localisée dans l'emprise de l'ancien fossé, ses fondations présentent une mise en œuvre particulière. Malgré une importante campagne de récupération des maçonneries, il est possible de restituer des structures profondément ancrées sur caissons de forme subcirculaire. Leur diamètre moyen est d'environ 3,50 m pour une profondeur supérieure à $2,20 \mathrm{~m}$ [ill. 3]. Malgré l'abondante

iconographie concernant ce secteur, aucune représentation de ce bâtiment ne nous est parvenue. L'analyse stratigraphique a cependant montré que sa construction n'intervient qu'à la suite d'une des deux phases d'incendie que la fouille a mis en évidence, correspondant certainement aux événements précités. Ce bâtiment est probablement détruit lors du deuxième incendie, moins de deux ans plus tard, ce qui explique pourquoi il n'apparait sur aucun document, qu'il s'agisse du cadastre ou sur des tableaux et estampes.

Si la chronologie de l'édifice est assez bien cernée, une piste peut être proposée pour sa destination. Ses tranchées de récupération étaient comblées par d'importantes quantités de briques ayant subi une forte exposition au feu, allant jusqu'à la vitrification pour certaines. Elles proviennent sans aucun doute d'une structure de chauffe. Il pourrait s'agir de la pompe à feu mais un texte nous précise qu'elle est bâtie en pierre de taille. Il reste alors l'hypothèse des fours à pain industriels. La puissance des fondations du bâtiment et la mise en œuvre adoptée démontre en premier lieu la volonté d'assoir l'édifice - imposant? - et de lui assurer une stabilité suffisante puisque construit dans l'ancien fossé. Cependant la présence des caissons circulaires pourrait faire écho, en négatif, à la présence des fours situés juste au-dessus. Après 1860, les
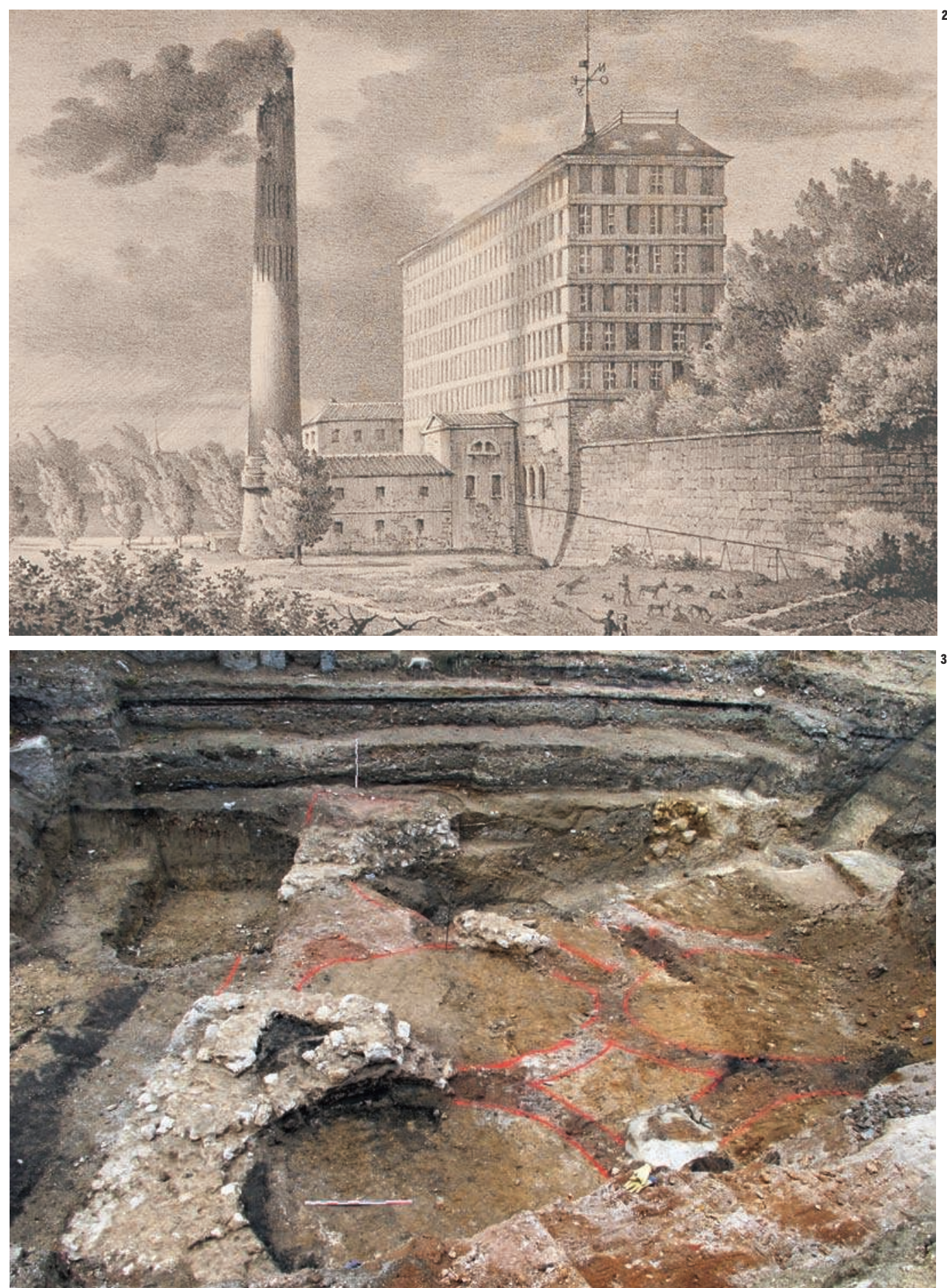

terrains sont un temps la propriété des hospices d'Orléans avant qu'une école d'artillerie n'y soit installée en 1874 .

\section{Références bibliographiques}

Daumas M., 1980, L'archéologie industrielle en France, Paris, Robert Laffont, $463 \mathrm{p}$.

Robert H., 2008, «La Motte Sanguin d'Orléans : de la filature de coton à la minoterie, une aventure industrielle », Bulletin de la Société Archéologique et Historique d'Orléans, 157, p. 5-48.

JesSET S., 2005, Orléans, «La Motte Sanguin " : Loiret-Centre. Rapport de diagnostic, Pantin, Inrap CIF, 3 vol., 117 p., 73 p. et 13 p.
2. Vue du nord de la manufacture de coto au début du XIXe $\mathbf{s}$. depuis la contrescarpe du foss de ville. On observe à droite l'élévation conservée de la terrasse d'artillerie, alors qu'à l'arrière la courtine a été totalement détruite Au dernier plan, derrière la rangée d'arbres : la Loire (MHO 72-5-11).
3. Le bâtiment sur caissons, vu du sud. On observera, sur la gauche du cliché et sur la berme inférieure à l'arrière-plan, le sédiment noirâtre issu du premier incendie et coupé par le creusement de la tranché de fondation de l'édifice. La phase du second incendie est nettement visible sur la berme supérieure. 